\title{
Critical Exponents from Non-Linear Functional Equations for Partially Directed Cluster Models
}

\author{
T. Prellberg and R. Brak * \\ Department of Mathematics, The University of Melbourne \\ Parkville, Victoria 3052, Australia
}

July 16, 2001

\begin{abstract}
We present a method for the derivation of the generating function and computation of critical exponents for several cluster models (staircase, bar-graph, and directed columnconvex polygons, as well as partially directed self-avoiding walks), starting with nonlinear functional equations for the generating function. By linearising these equations, we first give a derivation of the generating functions. The non-linear equations are further used to compute the thermodynamic critical exponents via a formal perturbation ansatz. Alternatively, taking the continuum limit leads to non-linear differential equations, from which one can extract the scaling function. We find that all the above models are in the same universality class with exponents: $\gamma_{u}=-1 / 2, \gamma_{t}=-1 / 3$ and $\phi=2 / 3$. All models have as their scaling function the logarithmic derivative of the Airy function.
\end{abstract}

PACS numbers: 05.50.+q, 05.70.fh, 61.41.+e

Key words: Functional Equations, Cluster models, Polygons, Critical Exponents, Scaling functions, Non-linear differential equation.

\footnotetext{
*email: prel, brak@mundoe.maths.mu.oz.au
} 


\section{Introduction}

Steady progress is being made in the mathematical analysis of certain geometric cluster models. These objects can be considered as combinatorial objects to be enumerated, or as models of physical systems such as vesicles or polymers. A selection of these models is illustrated in figure 1. Traditionally only the square lattice objects have been studied, although the generalisation to other lattices is straightforward. Combinatorialists have generally studied the polygon models and call these objects polyominoes. A short review of the history of the solutions of the various types of polyominoes can be found in M. Delest [1].

The two-variable generating functions for the area and perimeter (polygon models), or length and interactions (walk models), have been solved by a variety of methods. It is frequently possible to prove a bijection between words of an algebraic language and the combinatorial objects of interest [2], an idea going back to Schützenberger [3]. An alternative method of solution involving recurrence relations goes back to Temperley [4]. This method has been subsequently extended to solve several more models $[5,6,7,8]$. Some of the walk models can also be partially solved using transfer matrix techniques [9].

All these models possess the characteristic feature that their single variable generating functions are algebraic, whilst the two variable generating functions are expressed in terms of $q$-series. These are generally $q$-Bessel functions [10,11] or $q$-hypergeometric functions [12]. The $q$-series solutions to this class of models have been obtained by several techniques. A method developed by Temperley [4] leads to a recurrence relation which is solved by a $q$ series [5]. The recurrence relation is directly related to a linear functional equation which can also be solved by $q$-series methods [8]. In algebraic language theory the $q$-series appear as $q$-extensions or $q$-grammars [13].

We will use the language and notation of the polygon models in what follows, but mutatis mutandis the same results apply for the walk models.

The $q$-series solutions are satisfactory representations of the combinatorial problem in that the numbers of configurations of a given length and area can be computed in polynomial time (compared with the exponential time required for explicit enumeration). However, it is still not possible to easily extract the asymptotic behaviour of the generating function from the $q$-series. 


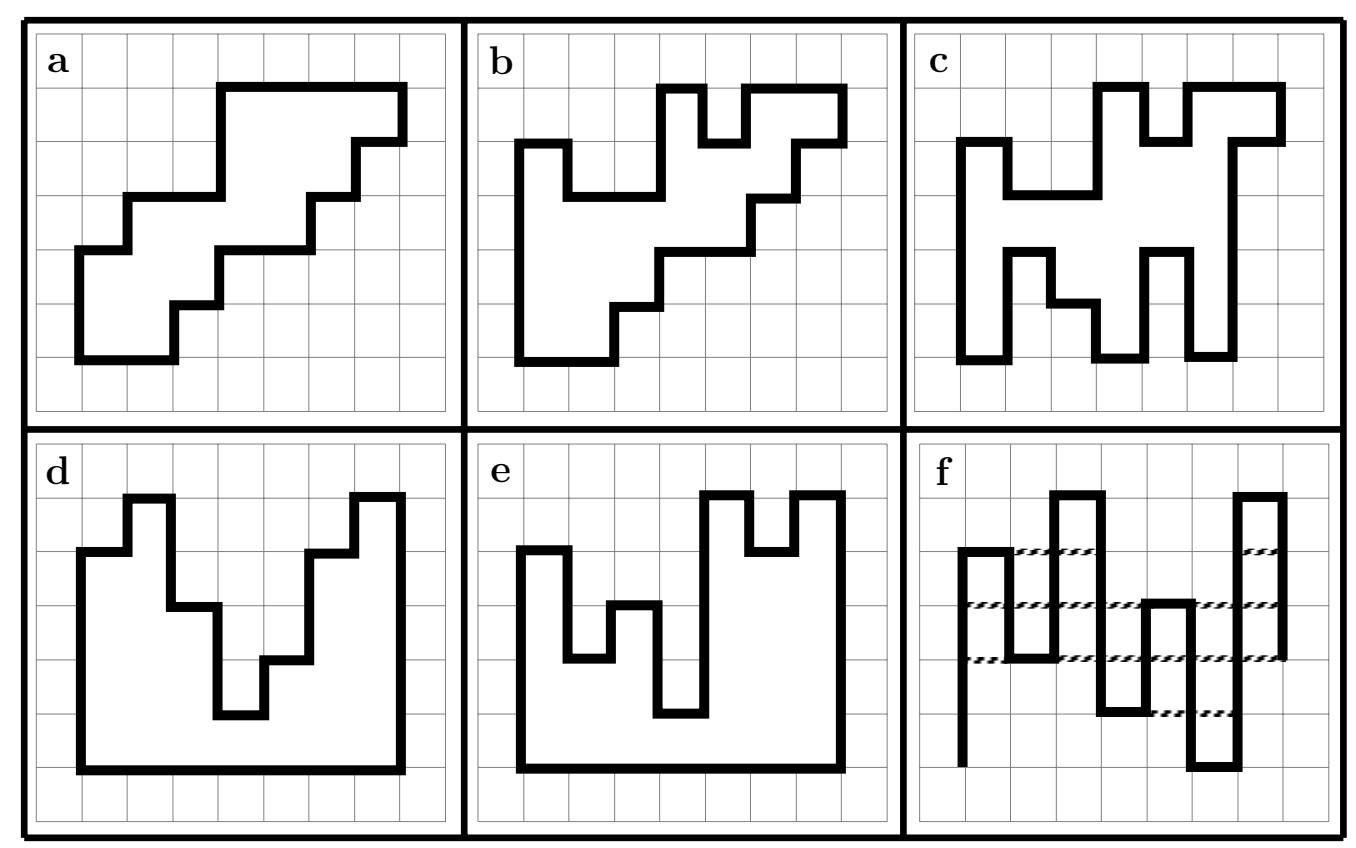

Figure 1: Typical configurations for a selection of directed geometric cluster models. a) staircase polygons, b) directed column-convex polygons, c) column-convex polygons, d) bargraph polygons, e) alternating bar-graph polygons and f) ZL walks

The principal purpose of this paper is to 'solve' this asymptotic problem without a direct assault on the $q$-functions. If only the asymptotics of the generating function is required then a direct assault on the $q$-functions is a wasted effort as a large part of the leading asymptotic function cancels and only the subdominant factors contribute to the generating function.

We approach the asymptotic problem using two different methods: i) A formal perturbation theory. This method has the advantage of giving several critical exponents for the lattice models. For this, the asymptotic form has to be assumed in order to identify the exponents. ii) Non-linear differential equations. This method has the advantage of deriving the asymptotic function and hence the critical exponents exactly, however it only applies to the semi-continuous version of the lattice model. If one accepts that the continuous version of a model has the same asymptotic behaviour as the lattice model then of course one has the lattice exponents as well.

We now outline the general form of the asymptotic behaviour of this class of models 
(for more details see Brak et al [14]). The principal object of study is the area-perimeter generating function $G(y, q)$, defined as

$$
G(y, q)=\sum_{m=1}^{\infty} A_{m}(y) q^{m}=\sum_{n=2}^{\infty} P_{2 n}(q) y^{n},
$$

where

$$
A_{m}(y)=\sum_{n=2}^{\infty} c_{m}^{2 n} y^{n}, \quad P_{2 n}(q)=\sum_{m=1}^{\infty} c_{m}^{2 n} q^{m},
$$

and $c_{m}^{2 n}$ is the number of polygons of the required type with perimeter $2 n$ and area $m$. Thus, $A_{m}(y)$ and $P_{2 n}(q)$ are the generating functions for polygons with a fixed area, respectively perimeter.

If we consider $q$ as a parameter and $y$ the variable, then $G$ is a series in $y$ with coefficients $P_{2 n}(q)$. If $G$ converges then its radius of convergence, $y_{c}(q)$ is given by

$$
y_{c}(q)=\lim _{n \rightarrow \infty} P_{2 n}(q)^{-\frac{1}{n}}
$$

It is usually straightforward to prove the existence of $y_{c}(q)$ using super- or submultiplicative inequalities $[15,6]$. For the polygon models a plot of the radius of convergence $y_{c}(q)$ as a function of $q$ is of the general form shown in figure 2. Alternatively one can fix $y$ and consider $G$ as a function of $q$ with radius of convergence $q_{c}(y)$. Then for polygon models the generating function is singular along the line $q=1$ between $y=0$ and some point $y_{t}=y_{c}(1)$. For other models this line may be more complicated. The point $\left(q_{t}, y_{t}\right)$ is an example of a "tricritical" point [14] where $q_{t}=q_{c}\left(y_{t}\right)$. For polygon models $q_{t}=1$. Elucidating the singularity structure of the generating function around this point is the principal interest of this paper.

When $q=1, G$ only generates the polygons by perimeter. For all the above models $G(y, 1)$ is an algebraic function and hence has a branch point with exponent $\gamma_{u}$ at $y_{t}$, that is

$$
G(y, 1) \sim A\left(y_{t}-y\right)^{-\gamma_{u}}, \quad y \rightarrow y_{t}^{-}
$$

For $y=y_{t}$, the generating function has a branch point-like singularity in $q$ at $q_{t}$, but with a different exponent, $\gamma_{t}$ where $\gamma_{t}$ is defined through

$$
G\left(y_{t}, q\right) \sim B\left(q_{t}-q\right)^{-\gamma_{t}}, \quad q \rightarrow q_{t}^{-}
$$

These two different asymptotic behaviours can be combined into a scaling function $f$, where

$$
G(y, q) \sim\left(q_{t}-q\right)^{-\gamma_{t}} f\left(\left\{q_{t}-q\right\}^{-\phi}\left\{y_{t}-y\right\}\right)
$$




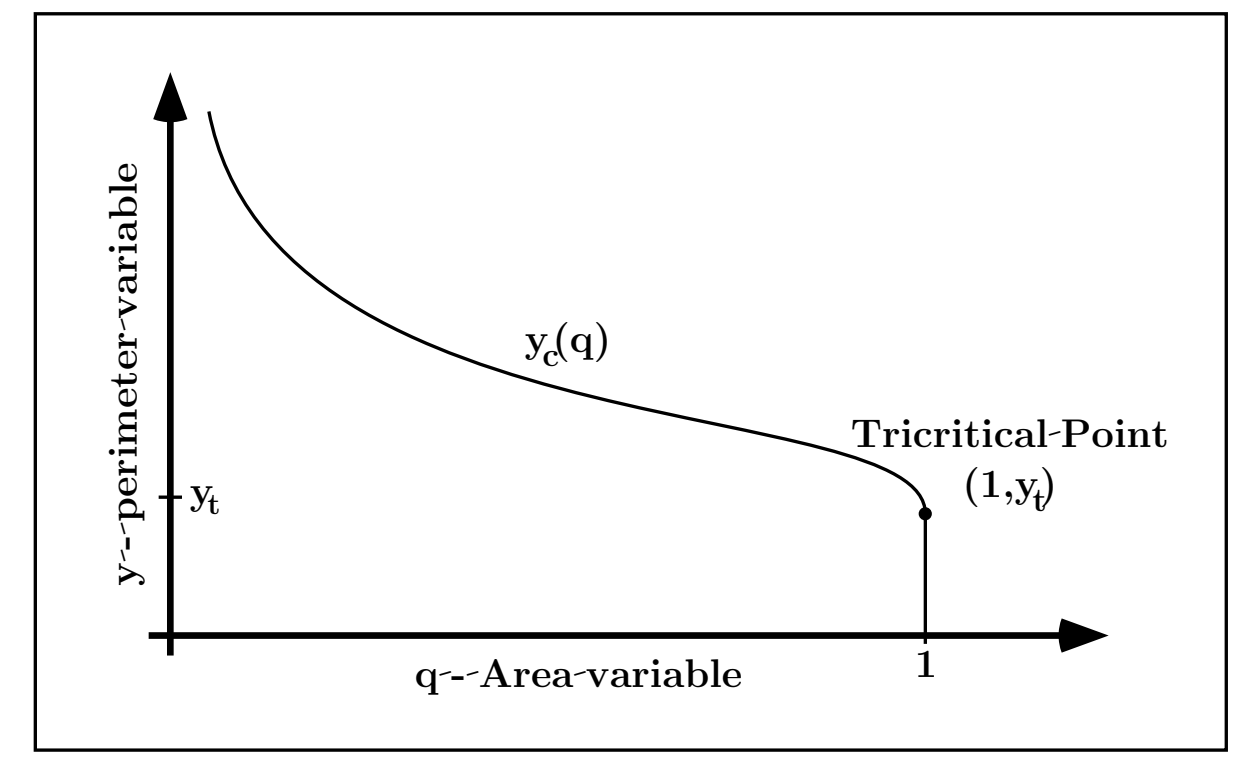

Figure 2: The schematic form of the radius of convergence of the area-perimeter generating function for the polygon models

with

$$
f(z) \sim \begin{cases}z^{-\gamma_{u}} & \text { if } z \rightarrow \infty \\ 1 & \text { if } z \rightarrow 0^{+} .\end{cases}
$$

where $\phi$ is called the tricritical crossover exponent and $\gamma_{u}=\gamma_{t} / \phi$.

What exactly does (1.6) mean? The righthand side is the asymptotic behaviour of $G$ as $q \rightarrow q_{t}^{-}$but is uniform in the variable $y$ in some neighbourhood of $y_{t}$. The uniformity is essential, as it enables one to interchange the order of the two limits $q \rightarrow q_{t}^{-}$and $y \rightarrow y_{t}^{-}$ and hence obtain the two different asymptotic behaviours (1.4) and (1.5). This uniformity will be explicitly shown for the staircase model.

One further behaviour of $G$ is of interest: the shape of the $y_{c}(q)$ curve in the neighbourhood of the tricritical point. For these polygon models, unlike some walk models, it is just a straight line for $y_{c}(q)<y_{t}$, however for $y_{c}(q)>y_{t}$ it is expected that

$$
y_{c}(q)-y_{t} \sim\left(q_{t}-q\right)^{1 / \psi}, \quad q \rightarrow q_{t}^{-}
$$

with a shape exponent $\psi$ which is related to the crossover exponent $\phi$ via $\psi=1 / \phi[14]$.

Considerable success at calculating the critical exponents was achieved by solving a semicontinuous model related to the lattice model. In particular, the ZL walk model, where the 
configurations are alternating partially directed self-avoiding walks [16], has been solved on the lattice [8] and in semi-continuum [16]. The semi-continuous model is exactly the same as the lattice model except that the length of the vertical steps of the walk can take any positive real value instead of being constrained to integer values. The exponents for the semi-continuous model are easily computed for ZL walks, as the solution is expressible in terms of Bessel functions whose asymptotics are well documented. For the lattice model the situation is quite different. Here the solution is expressible in terms of $q$-Bessel functions, and very little is known about their asymptotics. Because of this problem we have concentrated on obtaining the asymptotics along a route that does not depend on the $q$-Bessel functions. By using a formal perturbation expansion it has been shown in the case of IPDSAW [8] that the exponents for the lattice model and the semi-continuous model are exactly the same. Furthermore, renormalisation group arguments show that a lattice model and its semicontinuous version should have the same asymptotic behaviour. Accepting this argument means that it is sufficient to find the asymptotic behaviour of the semi-continuous model to have it for the lattice model as well.

It has also been shown for the ZL walk model that the semi-continuous model can be obtained from the lattice model by taking the continuum limit [8]. The continuum limit for this model corresponds to letting the lattice spacing in the vertical direction tend to zero whilst the 'physical' height is held fixed. In this limit the $q$-Bessel functions of the lattice model become the Bessel functions of the semi-continuous model [8]. In this sense one can think of the lattice model as the $q$-extension of the semi-continuous model.

The idea of using the continuum limit to compute the critical exponents is extended in this paper. The essential new ingredient of this paper is the starting point. Previous solutions have been obtained via recurrence relations or equivalently via linear functional equations. In this paper we use non-linear functional equations. This form of the functional equation is more suited to the limit $q \rightarrow q_{t}$.

We will show that the generating function for all the models in this class satisfy a quadratically non-linear functional equation, or set of coupled non-linear functional equations. All the non-linear functional equations obtained in this paper are solved by a linearising transformation. The linear functional equation can then by solved with a $q$-series Ansatz. This gives a new and elegant derivation of results obtained elsewhere [22]. 
Starting from the non-linear functional equation we take the continuum limit. This gives rise to a non-linear differential equation or a coupled set of non-linear differential equations. Solving the differential equation gives the solution to the semi-continuous version. For the all the models we study in this paper the differential equation is a generalised Riccati equation[17]. A $k$-th order generalised Riccati equation can be linearised by a simple transformation and always lead to a solution of the form

$$
g(t)=\frac{\sum_{i=1}^{k} c_{i} w_{i}^{\prime}(t)}{\sum_{i=1}^{k} c_{i} w_{i}(t)},
$$

where $w_{i}(t)$ are solutions of the linearised equation (the prime denotes differentiation). Even though the column-convex model does not appear to be of an exact Riccati form (1.9) [18], it is amenable to the methods presented here.

Having obtained the non-linear differential equation one has two immediate methods to obtain the asymptotic behaviour. Either one attempts to solve the equation explicitly and extracts the asymptotics from the resulting solutions, or one works directly with the nonlinear differential equation. For the simpler models we will use both methods whilst for the more complex models we use only the latter method.

The asymptotics can be obtained directly from the differential equation by using the method of dominant balance [19]. We show that asymptotically the non-linear differential equations for all the models have a generalised homogeneous or scaling solution. The scaling function (i.e. $f(z)$ of $(1.7)$ ) satisfies a Riccati equation of the form

$$
\frac{d f}{d z}=c f^{2}-b z
$$

where $b$ and $c$ are model dependent constants. This equation can be linearised by the transformation

$$
f(z)=-\frac{1}{c h(z)} \frac{d h}{d z}
$$

if $h(z)$ satisfies the linear equation

$$
\frac{d^{2} h}{d z^{2}}-b c z h(z)=0
$$

This is the Airy equation. Thus, after changing back to the original variables and inserting initial conditions, we find that asymptotically all the area-perimeter generating functions 
have the scaling function

$$
f(z)=+\left(\frac{b}{c^{2}}\right)^{1 / 3} \frac{\mathrm{Ai}^{\prime}\left(\{c b\}^{1 / 3} z\right)}{\operatorname{Ai}\left(\{c b\}^{1 / 3} z\right)}
$$

and the critical exponents are

$$
\gamma_{u}=-\frac{1}{2} \quad \gamma_{t}=-\frac{1}{3} \quad \phi=\frac{2}{3} \quad \psi=\frac{3}{2} .
$$

\section{Models}

The particular models we consider are subsets of column-convex polygons. A polygon is called column-convex if the intersection with any vertical line is convex, i.e. the intersection consists of only one connected line segment. Equivalently, column-convex polygons are generated by two mutually self-avoiding partially directed self-avoiding walks with common start and end points. (Partially directed walks are self-avoiding walks in which no steps into the negative $x$ direction are allowed.) By putting several restrictions on these walks, we can define various models (see figure 1).

If the lower and upper walks are fully directed (i.e. only steps into the positive $x$ and $y$ directions are allowed), we get the model of staircase polygons $S$ (figure 1a).

If the upper walk is partially directed and the lower walk is fully directed, we get the model of directed column-convex polygons $D$ (figure 1b). If both walks are partially directed without restrictions, we get the model of column-convex polygons (figure 1c).

If the upper walk is partially directed and the lower walk is restricted to be horizontal, we get the model of bar-graph polygons $B$ (figure 1d). A particularly simple subset is given by the additional restriction that the upper walk reverse direction after every horizontal step, leading to alternating bar-graph polygons $R$ (figure 1e).

For each of these models, we extend the definition of the generating function from the introduction as follows. Let $c_{m}^{n_{x}, n_{y}}$ be the number of polygons with $2 n_{x}$ horizontal steps and $2 n_{y}$ vertical steps which enclose an area of size $m$. (Clearly the numbers of horizontal and vertical steps are even.) We then define the polygon generating function $G(x, y, q)$ to be

$$
G(x, y, q)=\sum c_{m}^{n_{x}, n_{y}} x^{n_{x}} y^{n_{y}} q^{m} .
$$

We further consider models of interacting partially directed self-avoiding walks (IPDSAW) which are enumerated with respect to their length and the number of nearest-neighbour 
bonds. A particular subset is given by walks which reverse direction after every horizontal step (ZL), see figure 1f.

Let $c_{m}^{n_{x}, n_{y}}$ be the number of walks with $n_{x}$ horizontal and $n_{y}$ vertical steps and $m$ the number of nearest-neighbour bonds. Then we define the walk generating function

$$
H(x, y, \omega)=\sum c_{m}^{n_{x}, n_{y}} x^{n_{x}} y^{n_{y}} \omega^{m}
$$

\section{$3 \quad$ Functional Equations}

The technique for solving these models is to use a geometrical partition of the set of all polygons into disjoint subsets. These subsets are chosen such that they enable one to give construction rules that can be transformed into equations for the corresponding generating functions. A related method for the derivation of functional equations based on the theory of algebraic languages has been developed in $[20,21]$.

These equations can then in principle be used to solve for the generating function. As we shall see, one can also extract information about the singularity structure of the generating function directly from the functional equations.

We start this section by presenting an example with a particularly simple partition to illustrate the method. In general, the partition will be more complicated. The partitions of all of the following models have, however, one feature in common with this example. One set of the partition will be a set $P_{1}$ of "inflated" polygons, i.e. polygons which are generated from the set $P$ of all polygons by increasing the height of each column by one (replacement of $x$ by $q x$ ) and thereby increasing the number of vertical perimeter bonds by 2 (multiplication by $y$ ), leading to the equation

$$
P_{1}(x, y, q)=P(q x, y, q) y
$$

To start with the example, assume that we would like to describe the generating function $C(x, y, q)$ for the set $C$ of all columns of width 1 . In an abuse of notation, we will use the same symbol for a set and its associated generating function. A partition of the set $C$ of all columns into two sets is given by collecting all columns of height larger than 1 into the set $C_{1}$, leaving the set $C_{2}=C \backslash C_{1}$ which contains only the unit square. The set $C_{1}$ can easily 
$\underline{\text { Columns }}$

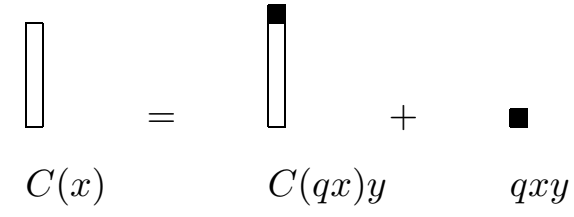

Figure 3: The diagrammatic form of the functional equation for single columns together with its generating function representation

be seen to be constructed from the set of all columns by increasing their height by one area element. This is shown symbolically in figure 3 .

In terms of generating functions, we can write this as

$$
C_{1}(x, y, q)=C(q x, y, q) y .
$$

The generating function for $C_{2}$ is simply

$$
C_{2}(x, y, q)=q x y,
$$

and summing up $C=C_{1}+C_{2}$ leads to a functional equation for $C$,

$$
C(x, y, q)=C(q x, y, q) y+q x y
$$

which can be immediately solved by iteration to give

$$
C(x, y, q)=\frac{q x y}{1-q y} .
$$

\section{Bar-graph polygons}

Our next example will be bar-graph polygons, that is column-convex polygons with a horizontal lower boundary. We can partition the set $B$ of all bar-graph polygons by first splitting off the set $B_{1}$ of inflated bar-graph polygons. If $B(x, y, q)$ denotes the generating function for the set of all bar-graph polygons, then the generating function $B_{1}(x, y, q)$ of the subset $B_{1}$ is given as

$$
B_{1}(x, y, q)=B(q x, y, q) y
$$


$\underline{\text { Alternating Bar-graph Polygons }}$

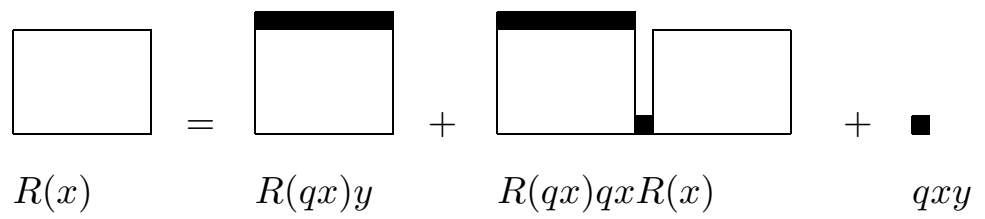

Bar-graph Polygons
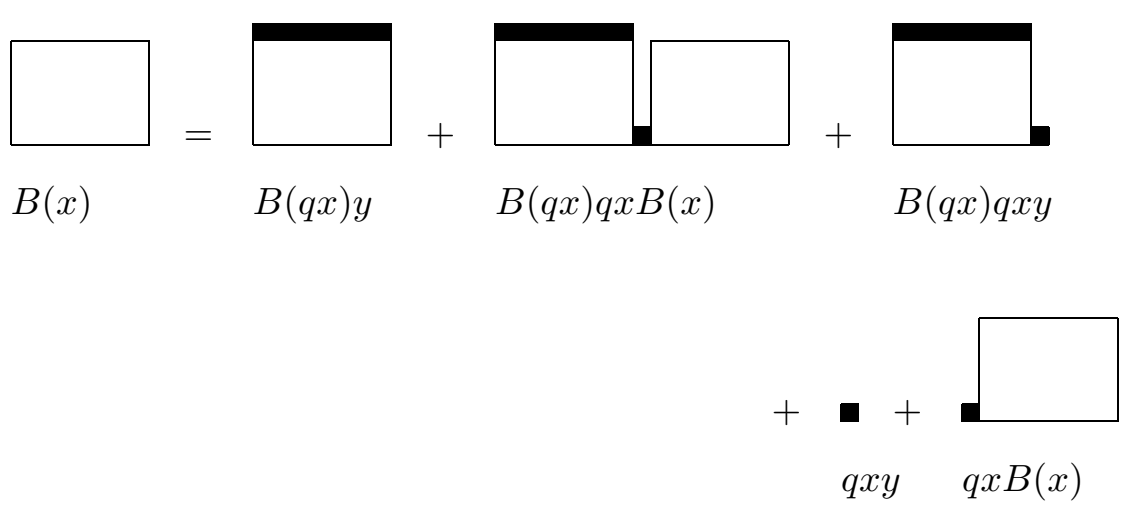

Figure 4: The diagrammatic form of the functional equations for bar-graph polygons and alternating bar-graph polygons

The further decomposition is shown symbolically in figure 4 . The remaining bar-graph polygons in the set $B \backslash B_{1}$ have at least one column of height 1 . In general, to the left of the leftmost column of height 1 there is an inflated bar-graph polygon from the set $B_{1}$, whereas to the right of this column the remaining bar-graph polygon is a simple bar-graph polygon from the set $B$. We now define the set $B_{2}$ to be the set of bar-graph polygons generated by concatenating inflated bar-graph polygons with a bar-graph polygon to the right by a single column of height 1 . The generating function for the set $B_{2}$ therefore fulfills

$$
B_{2}(x, y, q)=B_{1}(x, y, q) y^{-1} q x B(x, y, q)=B(q x, y, q) q x B(x, y, q)
$$

where division by $y$ takes care of the reduction of perimeter due to concatenation. The remaining bar-graph polygons in the set $B \backslash\left(B_{1} \cup B_{2}\right)$ have the property that the leftmost 
column of height 1 is at the right or left end of the polygon, symbolised by the remaining configurations in figure 4 . They can be uniquely partitioned into the set $B_{3}$ of bar-graph polygons generated by concatenation of inflated bar-graph polygons with a single square to the right, the set $B_{4}$ containing only the single square, and the set $B_{5}$ of bar-graph polygons generated by concatenation of bar-graph polygons with a single square to the left, leading to the following equations for the respective generating functions

$$
\begin{aligned}
& B_{3}(x, y, q)=B(q x, y, q) q x y \\
& B_{4}(x, y, q)=q x y \\
& B_{5}(x, y, q)=q x B(x, y, q) .
\end{aligned}
$$

Thus, we have partitioned the set $B$ of all bar-graph polygons into five disjoint subsets. Therefore the generating function for $B$ is the sum of the individual generating functions, $B=B_{1}+B_{2}+B_{3}+B_{4}+B_{5}$, and we get the functional equation

$$
B(x, y, q)=B(q x, y, q) y+\{1+B(q x, y, q)\} q x\{y+B(x, y, q)\}
$$

This equation can be solved for $B(x, y, q)$ and leads to a continued fraction representation of the generating function, given by iteration of

$$
B(x, y, q)=\frac{y}{q x}\left\{\frac{1}{1-q x-q x B(q x, y, q)}-1-q x\right\} .
$$

At this point one can of course easily generate related models, for example, the set of alternating bar-graph polygons (the subset of bar-graph polygons with an upper boundary whose vertical components alternate in direction) can be obtained by prohibiting the concatenations which lead to the sets $B_{3}$ and $B_{5}$ because these concatenations generate the non-alternating segments in the upper boundary. Denoting the generating function by $R(x, y, q)$, we therefore get immediately

$$
R(x, y, q)=R(q x, y, q) y+R(q x, y, q) q x R(x, y, q)+q x y
$$

which also leads to a continued fraction expansion.

\section{Staircase polygons}

For staircase polygons, one can employ a rather similar method of partitioning (see figure 5). Here it is more convenient to consider the overlap between neighbouring columns rather 
$\underline{\text { Staircase Polygons }}$

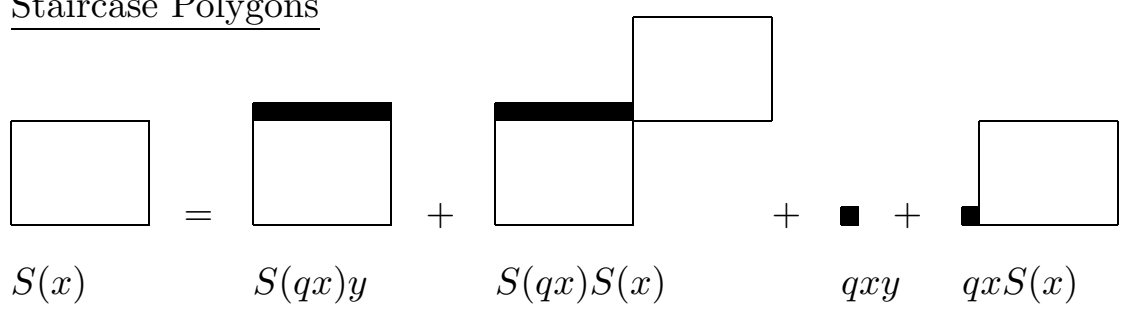

Directed Column-Convex Polygons
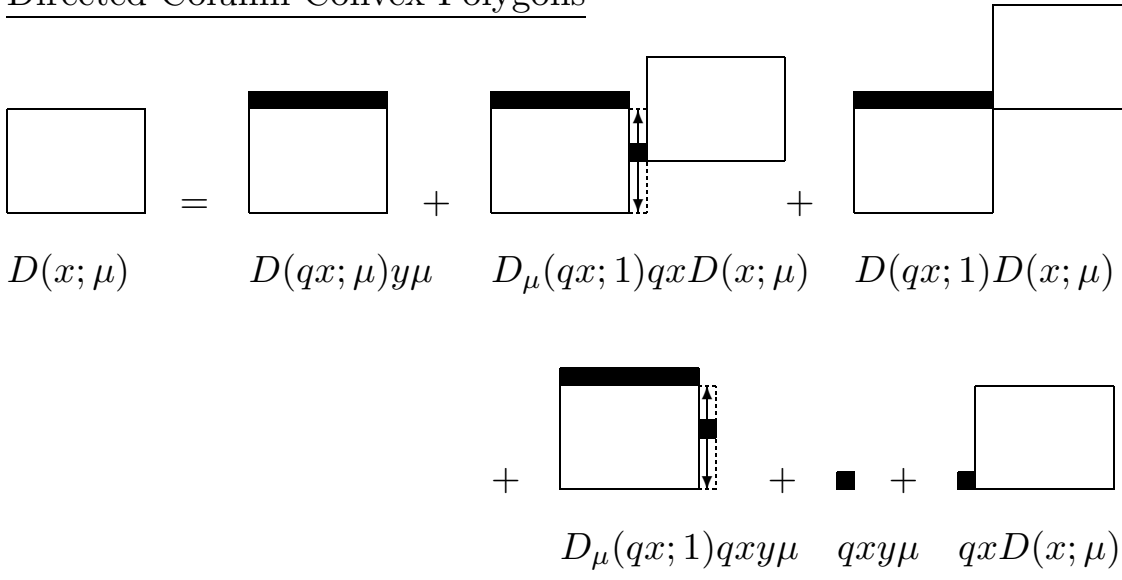

Figure 5: The diagrammatic form of the functional equations for staircase polygons and directed column-convex polygons

than the height of the columns, as the columns can be shifted against each other. One again splits the set $S$ up into the set $S_{1}$ of inflated staircase polygons and the set $S \backslash S_{1}$ of the remaining staircase polygons. These polygons share the property that they have at least one overlap of height 1 , so that we can consider the leftmost overlap of height 1 . Generally, this overlap splits the staircase polygon into two parts, an inflated one $\left(S_{1}\right)$ and a regular one $(S)$. Directedness of the staircase polygon ensures that these parts are joined by their respective corners. We therefore define the set $S_{2}$ to consist of those staircase polygons that are generated by concatenating to the right all inflated polygons of the set $S_{1}$ with all polygons of the set $S$ by their respective corners. The remaining set $S \backslash\left(S_{1} \cup S_{2}\right)$ contains degenerate cases which arise when the height of the first column is 1 (see figure 5 for details). 
The complete partition leads to the functional equation

$$
S(x, y, q)=\{S(q x, y, q)+q x\}\{y+S(x, y, q)\}
$$

(this is exactly equation (6.1) in [24], where it has been derived using Schützenberger's methodology).

\section{Directed column-convex polygons}

We now turn to a more complex model, directed column-convex polygons [21]. The additional degree of freedom in the construction necessitates the introduction of another variable in the generating function. As in the case of staircase polygons, the set $D$ of all directed columnconvex polygons is partitioned by first splitting off the set $D_{1}$ of inflated directed columnconvex polygons. The set $D \backslash D_{1}$ of the remaining polygons is further partitioned by again considering the leftmost overlap of two neighbouring columns of height 1. As above, this overlap splits the polygon into two parts. To the left of the overlap is an inflated directed column-convex polygon $\left(D_{1}\right)$ which is joined to another polygon $(D)$ by a single square. This single square is attached to the bottom corner of the right polygon but can be joined to the inflated polygon on the left everywhere along its right height. We define the set $D_{2}$ to be the set of all directed column-convex polygons generated in just such a way, however we exclude the case when the single square is attached to the inflated polygon in the top-most position. Here the polygons can be joined directly as was the case for staircase polygons, leading to the set $D_{3}$. Naturally, we also have a few degenerate cases, which we partition further into the sets $D_{4}, D_{5}$, and $D_{6}$ (again, see figure 5 for details).

In order to write down a functional equation for this model, we need to keep track of the height $r$ of the rightmost column of these polygons. We write

$$
c_{m}^{n_{x}, n_{y}}=\sum_{r=1}^{\infty} c_{m}^{n_{x}, n_{y}, r}
$$

and define

$$
D(x, y, q ; \mu)=\sum c_{m}^{n_{x}, n_{y}, r} x^{n_{x}} y^{n_{y}} q^{m} \mu^{r} .
$$

The first term in the functional equation for $D$ is again given by inflation

$$
D_{1}(x, y, q ; \mu)=D(q x, y, q ; \mu) y \mu .
$$


The next term describes the concatenation of an inflated polygon to another one. There is a multiplicity due to the arbitrary position of the middle square with respect to the left polygon (i.e. if the height of the left column is $r$ units then the single square can be attached at $r-1$ locations). This gives rise to a factor of $r-1$ in the generating function and thus can be written as

$$
\left.\frac{\partial}{\partial \mu} \frac{1}{\mu} D_{1}(x, y, q ; \mu)\right|_{\mu=1}=\left.\frac{\partial}{\partial \mu} D(q x, y, q ; \mu) y\right|_{\mu=1}=D_{\mu}(q x, y, q ; 1) y
$$

where we denote differentiation with respect to the parameter $\mu$ with a subscript. The generating function for the second diagram can therefore be written as

$$
D_{2}(x, y, q ; \mu)=D_{\mu}(q x, y, q ; 1) q x D(x, y, q ; \mu) .
$$

If the polygons get joined directly at the corners, we set $\mu=1$ for the left polygon and write

$$
D_{3}(x, y, q ; \mu)=D(q x, y, q ; 1) D(x, y, q ; \mu) .
$$

The remaining terms can be written as

$$
\begin{aligned}
& D_{4}(x, y, q ; \mu)=D_{\mu}(q x, y, q ; 1) q x y \mu \\
& D_{5}(x, y, q ; \mu)=q x y \mu \\
& D_{6}(x, y, q ; \mu)=q x D(x, y, q ; \mu)
\end{aligned}
$$

and summing up $D=D_{1}+D_{2}+D_{3}+D_{4}+D_{5}+D_{6}$ leads to the

$$
\begin{aligned}
D(x, y, q ; \mu)= & \left\{1+D_{\mu}(q x, y, q ; 1)\right\} q x\{y \mu+D(x, y, q ; \mu)\} \\
& +D(q x, y, q ; \mu) y \mu+D(q x, y, q ; 1) D(x, y, q ; \mu)
\end{aligned}
$$

We can transform this functional-differential equation to a set of functional equations by partially differentiating (3.25) with respect to $\mu$ and setting $\mu=1$. This leads to

$$
\begin{aligned}
d & =\left\{1+D_{\mu}\right\} q x\{y+d\}+D\{y+d\} \\
d_{\mu} & =\left\{1+D_{\mu}\right\} q x\left\{y+d_{\mu}\right\}+D\left\{y+d_{\mu}\right\}+D_{\mu} y
\end{aligned}
$$

where now

$$
d(x, y, q)=D(x, y, q ; 1) \quad d_{\mu}(x, y, q)=D_{\mu}(x, y, q ; 1)
$$


and

$$
D(x, y, q)=d(q x, y, q) \quad D_{\mu}(x, y, q)=d_{\mu}(q x, y, q)
$$

We can simplify this system of two functional equations further to get one equation in $D(x)=$ $D(x, y, q ; 1)$,

$$
\begin{aligned}
0 & =D\left(q^{2} x\right) D(q x) D(x) \\
& +y D\left(q^{2} x\right) D(q x)+y D\left(q^{2} x\right) D(x)-(1+q) D(q x) D(x) \\
& +y^{2} D\left(q^{2} x\right)-y(1+q) D(q x)+q(1+q x(y-1)) D(x) \\
& +y q^{2} x(y-1)
\end{aligned}
$$

and in the next sections it is merely a matter of taste that we choose to continue to work with the system (3.25) instead.

\section{ZL-walks}

Finally, the functional equation for the ZL-walks is,

$$
H(x)=x y+x q H(x)+(1 / \omega+x y) H(q x)+x q H(x q) H(x)
$$

where $q=\omega y$. This functional equation is very similar to the bar-graph equation. In fact in the continuum limit the same form of differential equation is obtained leading to the same scaling function and critical exponents. The only real difference between the ZL-walks and the bar-graph polygons is the process of inflation. For ZL-walks the process corresponds to adding on edge to each vertical segment of the walk (as opposed to adding one square to each column of the polygon).

\section{Solution of the Functional Equations}

We now present an explicit solution of the above mentioned functional equations. First we note that the functional equations for bar-graph polygons, alternating bar-graph polygons, staircase polygons, and ZL-walks can all be linearised, as they are of the form

$$
G(x) G(q x)+a(x) G(x)+b(x) G(q x)+c(x)=0,
$$


which can be linearised by use of the transformation

$$
G(x)=\alpha \frac{H(q x)}{H(x)}-b(x),
$$

where $\alpha$ has to be chosen to match the initial condition. This leads to a linear functional equation in $H(x)$,

$$
\alpha^{2} H\left(q^{2} x\right)+\alpha[a(x)-b(q x)] H(q x)+[c(x)-a(x) b(x)] H(x)=0 .
$$

Due to the special structure of the coefficients in this equation, we can now give explicit solutions to each of the above models as well as to the model of directed column convex polygons. As we will encounter a similar equation below, we shall state the solution for this type of equation in a slightly more general way.

Assume that we have a linear functional equation of the form

$$
0=x H(q x)+\sum_{k=0}^{N} \alpha_{k} H\left(q^{k} x\right) \quad \text { with } \quad \sum_{k=0}^{N} \alpha_{k}=0 .
$$

with $\alpha_{k}$ independent of $x$. The solution of (4.4) which is regular at $x=0$ is then given by

$$
H(x)=\sum_{n=0}^{\infty} \frac{(-x)^{n} q^{\left(\begin{array}{c}
n \\
2
\end{array}\right)}}{\prod_{m=1}^{n} \Lambda\left(q^{m}\right)} \quad \text { with } \quad \Lambda(t)=\sum_{k=0}^{N} \alpha_{k} t^{k} .
$$

We reiterate that it is crucial that $\Lambda(1)=\sum_{k=0}^{N} \alpha_{k}=0$.

We now apply this method in the case of staircase polygons. We use the transformation

$$
S(x)=y\left(\frac{T(q x)}{T(x)}-1\right),
$$

as only the choice of $\alpha=y$ leads to the needed cancellation of the $x^{0}$ coefficient. This transforms equation (3.14) into

$$
0=y T\left(q^{2} x\right)+(q x-1-y) T(q x)+T(x) .
$$

Therefore, we have

$$
\Lambda(t)=\frac{1}{q}-\frac{1+y}{q} t+\frac{y}{q} t^{2} .
$$

The condition $\Lambda(1)=0$ holds, so that we can solve equation (4.8) using (4.5). We get the solution

$$
T(x)=\sum_{n=0}^{\infty} \frac{(-q x)^{n} q^{\left(\begin{array}{c}
n \\
2
\end{array}\right)}}{(q, q y ; q)_{n}},
$$


where we have used the $q$-product notation

$$
\left(x_{1}, x_{2}, \ldots, x_{k} ; q\right)_{n}=\prod_{m=0}^{n-1}\left(1-x_{1} q^{m}\right)\left(1-x_{2} q^{m}\right) \ldots\left(1-x_{k} q^{m}\right) .
$$

The function $T(x)=T(x, y, q)$ is a $q$-deformation of a Bessel function. This calculation easily generalises for the other models with quadratic functional equations.

In the case of directed column convex polygons, we have a "cubic" equation, and there is no general way of linearising it. Surprisingly, it turns out that the same transformation, i.e.

$$
D(x)=y\left(\frac{E(q x)}{E(x)}-1\right)
$$

transforms equation (3.28) into a linear one,

$$
y^{2} E\left(q^{3} x\right)-y[q+y+1] E\left(q^{2} x\right)+\left[y+q+q y+q^{2} x(y-1)\right] E(q x)-q E(x)=0 .
$$

Moreover, this linear equation is of the desired form (4.4). Using (4.5) results now in

$$
E(x)=\sum_{n=0}^{\infty} \frac{((y-1) q x)^{n} q^{\left(\begin{array}{c}
n \\
2
\end{array}\right)}}{(q, q y, y ; q)_{n}} .
$$

Transformations (4.6) and (4.11) are the discrete analogue of the transformation (5.14) used to linearise the non-linear Riccati equation. The method presented in this section thus gives new and simple derivations of results previously calculated via the Temperley method (which leads to linear functional equations with an auxiliary parameter [22]).

It is remarkable that both models have generating functions which can be expressed in the form

$$
G(x, y, q)=y\left(\frac{H(q x, y, q)}{H(x, y, q)}-1\right)
$$

where the function $H$ fulfills a linear functional equation In the case of staircase polygons, this structure can also be explained via a bijection with heaps, which indicates that in the case of directed column-convex polygons there might be a similar bijection [23].

Finally, it is worth mentioning that in [24] a related class of convex polygons (as opposed to column-convex) has been studied via systems of $q$-differential equations. There, the authors first looked at the associated differential equations (these are constructed in a purely formal way and don't have the continuum limit interpretation of the differential equations in this paper). Upon finding the solution of the differential equations, a $q$-analogue had to be "guessed". 


\section{Continuum Limit}

In this section we define the semi-continuous staircase polygon and semi-continuous directed column-convex polygon models. We use this definition to explicitly construct a transformation which will allow us to take the continuum limit of the lattice model and hence obtain its corresponding semi-continuous version. The precise details of this formal method of constructing the continuum will be model dependent.

Taking the continuum limit on the level of the lattice model functional equations, we find that they reduce to non-linear differential equations. From these equations we deduce the asymptotic behaviour of the two models either by solving the differential equation explicitly and working with the solution or by extracting the asymptotics directly from the equation.

The semi-continuous model corresponds to allowing the lengths of the vertical segments of the corresponding lattice model to take on positive real values rather than only positive integer values. Mathematically this can be achieved in two ways: either by writing the generating function immediately allowing for continuous length segments, or by inserting the lattice spacing, $a$, explicitly into the lattice generating function and then taking the limit $a \rightarrow 0$. We will illustrate these two equivalent approaches using the staircase model.

\section{Semi-continuous Staircase Model}

We can explicitly introduce the constraint using two length variables for each column of the staircase as shown in figure 6 . The semi-continuous generating function is then defined by

$$
\mathcal{S}(x ; \tau, \epsilon)=\sum_{n=1}^{\infty} x^{n} \int_{0}^{\infty} d r_{1} \int_{0}^{r_{1}} d s_{1} \cdots \int_{s_{j-1}}^{\infty} d r_{j} \int_{0}^{r_{j}} d s_{j} \cdots \int_{s_{n-1}}^{\infty} d r_{n} \exp \left(-\mathcal{H}_{n}\right)
$$

with

$$
\mathcal{H}_{n}=\sum_{i=1}^{n}\left\{\tau\left(r_{i}-s_{i-1}\right)+\epsilon r_{i}\right\}
$$

Note, there are two integrals for every column except for the last, and the limits on the first pair are slightly different to those on the intermediate pairs. This expression can be evaluated by Temperley's method [18].

On the other hand, the lattice generating function can be written in an analogous form

$$
S(x ; y, q)=\sum_{n=1}^{\infty} x^{n} \sum_{\ell_{1}=1}^{\infty} \sum_{m_{1}=1}^{\ell_{1}} \ldots \sum_{\ell_{j}=m_{j-1}}^{\infty} \sum_{m_{j}=1}^{\ell_{j}} \cdots \sum_{\ell_{n}=m_{n-1}}^{\infty} \mathcal{B}_{n}
$$




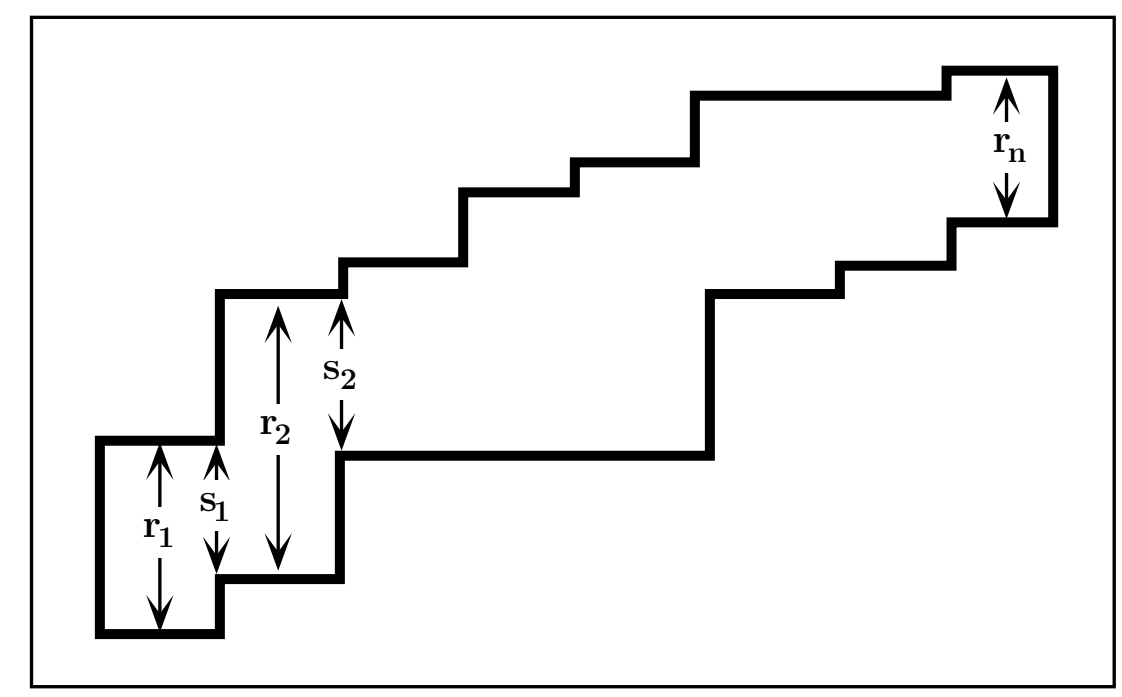

Figure 6: The continuous staircase polygon model showing the length variables

with

$$
\mathcal{B}_{n}=\prod_{i=1}^{n} y^{\ell_{i}-m_{i-1}} q^{\ell_{i}} .
$$

Clearly $\mathcal{B}_{n}$ is equivalent to $\mathcal{H}_{n}$ if we let $y=\exp (-\tau)$ and $q=\exp (-\epsilon)$ and consider $\ell$ and $m$ as continuous. This can be done explicitly as follows. Insert the vertical lattice spacing $a$ into the lattice generating function by replacing $x$ by $a^{2} x, y$ by $y^{a}$ and $q$ by $q^{a}$, so that $S(x, y, q)$ becomes

$$
S_{a}(x)=S\left(a^{2} x, y^{a}, q^{a}\right)
$$

This gives one factor of $a$ for each sum (with one to spare). Thus, as $a \rightarrow 0$ we then have

$$
\lim _{a \rightarrow 0} \frac{1}{a} S_{a}(x)=\mathcal{S}(x ; \tau, \epsilon) .
$$

Equation (5.6) is a transformation which takes the lattice model to the semi-continuous model and hence defines the "continuum limit" for the staircase model. Note that we have $S_{a}(x)=\mathrm{O}(a)$. The way the factors of $a$ have to be inserted varies from model to model and generally depends on the number of length variables associated with each column.

We now apply the above defined continuum limit to the staircase functional equation. The functional equation will become a non-linear differential equation. The process consists of two stages: first the lattice spacing is inserted explicitly, then the limit $a \rightarrow 0$ is taken. 
Thus, upon inserting the lattice spacing in the staircase model the functional equation (3.14) becomes

$$
S_{a}(x)=a^{2} x y^{a} q^{a}+a^{2} x q^{a} S_{a}(x)+y^{a} S_{a}\left(q^{a} x\right)+S_{a}\left(q^{a} x\right) S_{a}(x) .
$$

Letting $y=\exp (-\tau), q=\exp (-\epsilon)$ and using the result

$$
S_{a}\left(x e^{-a \epsilon}\right)=S_{a}(x)-\epsilon a x \frac{\partial}{\partial x} S_{a}(x)+\mathrm{O}\left(a^{2}\right)
$$

gives

$$
0=a^{2} x-\tau a S_{a}(x)+S_{a}(x)^{2}-\epsilon a x \frac{\partial}{\partial x} S_{a}(x)+\mathrm{O}\left(a^{3}\right) .
$$

Thus, dividing by $a^{2}$, taking the limit $a \rightarrow 0$, using (5.6), gives

$$
\lambda t \frac{\partial}{\partial t} \hat{\mathcal{S}}(t)=t-\hat{\mathcal{S}}(t)+\hat{\mathcal{S}}(t)^{2}
$$

where

$$
\lambda=\frac{\epsilon}{\tau}, \quad \quad \quad \quad \quad \text { and } \quad \hat{\mathcal{S}}(t)=\hat{x}(t ; \lambda)=\frac{1}{\tau} \mathcal{S}(x ; \tau, \epsilon) .
$$

This differential equation is a Riccati equation (with initial condition $\hat{\mathcal{S}}(0)=1$ ). It is easily solved for the perimeter only generating function (which corresponds to $q=1$, or equivalently, $\lambda=0)$ as the equation reduces to a quadratic whose solution is

$$
\hat{\mathcal{S}}(t ; \lambda=0)=\frac{1}{2}\{1-\sqrt{1-4 t}\} .
$$

Hence we see that the perimeter generating function has a square-root singularity at $t=1 / 4$ and thus we have

$$
\gamma_{u}=-\frac{1}{2} \quad \text { and } \quad t_{c}=\frac{1}{4} .
$$

For $\lambda \neq 0$ the Riccati equation can be solved by the substitution

$$
\hat{\mathcal{S}}=-\lambda t \frac{h^{\prime}(t)}{h(t)}
$$

where the prime denotes differentiation with respect to the functions argument. The function $h(t)$ satisfies the linear differential equation

$$
\lambda^{2} t h^{\prime \prime}+\lambda(\lambda+1) h^{\prime}+h=0
$$


A few simple changes of variable reduces this equation to Bessel's equation. The general solution is

$$
h(t)=t^{-\frac{1}{2 \lambda}}\left\{c_{1} J_{\frac{1}{\lambda}}(2 \sqrt{t} / \lambda)+c_{2} \mathrm{Y}_{\frac{1}{\lambda}}(2 \sqrt{t} / \lambda)\right\} .
$$

Inserting the initial condition and back substituting gives

$$
\hat{\mathcal{S}}=\frac{1}{2}+\sqrt{t} \frac{\mathrm{J}_{\frac{1}{\lambda}}^{\prime}(2 \sqrt{t} / \lambda)}{\mathrm{J}_{\frac{1}{\lambda}}(2 \sqrt{t} / \lambda)} .
$$

This is the explicit solution of the semi-continuous model. In section 7 we consider its asymptotic behaviour.

\section{Semi-continuous directed column-convex model}

For the directed column-convex polygons we have two coupled functional equations. An analogous expression to (5.1) for the semi-continuous generating function, $\mathcal{D}(x ; \tau, \epsilon)$, shows that

$$
d\left(a^{2} x ; y^{a}, q^{a}\right)=\mathrm{O}(a)
$$

but that

$$
d_{\mu}\left(a^{2} x ; y^{a}, q^{a}\right)=\mathrm{O}(1)
$$

Thus the continuum limit of (3.25) gives

$$
\begin{aligned}
\lambda t \frac{\partial}{\partial t} \hat{\mathcal{D}}(t) & =t\left\{1+\mathcal{D}_{\mu}(t)\right\}+\hat{\mathcal{D}}(t)^{2}-\hat{\mathcal{D}}(t) \\
\lambda t \frac{\partial}{\partial t} \mathcal{D}_{\mu}(t) & =\left\{1+\mathcal{D}_{\mu}(t)\right\}\{\hat{\mathcal{D}}(t)-1\}+1,
\end{aligned}
$$

where

$$
\lambda=\frac{\epsilon}{\tau} \quad t=\frac{x}{\tau^{2}} \quad \hat{\mathcal{D}}(t)=\frac{1}{\tau} \mathcal{D}(x ; \tau, \epsilon)
$$

and $\mathcal{D}_{\mu}(t)$ is the semi-continuous analogue of $D_{\mu}(x)$.

As for the staircase model, we can find the perimeter only generating function by putting $\lambda=0$. Eliminating $\mathcal{D}_{\mu}(t)$ from the two equations (5.18) and (5.19), gives a cubic equation for $\hat{\mathcal{F}}=\hat{\mathcal{D}}-1$, where

$$
\hat{\mathcal{F}}^{3}+\hat{\mathcal{F}}^{2}-t=0 \quad(\lambda=0) .
$$

For $t \rightarrow 4 / 27$, the relevant solution of (5.21) gives

$$
\hat{\mathcal{F}} \sim-\frac{2}{3}-\sqrt{\frac{4}{27}-t} .
$$


Thus the perimeter generating function for directed column-convex model has a square-root singularity at $t=4 / 27$, and hence

$$
\gamma_{u}=-\frac{1}{2} \quad t_{c}=\frac{4}{27}
$$

For $\lambda \neq 0, \mathcal{D}_{\mu}$ can be eliminated from the coupled equations (5.19) to give a single non-linear differential equation for $\mathcal{H}(t)=\hat{\mathcal{F}} / \lambda t$, where

$$
\mathcal{H}^{\prime \prime}+\left(-3 \mathcal{H}+\frac{2}{t}-\frac{1}{\lambda t}\right) \mathcal{H}^{\prime}+\mathcal{H}^{3}+\left(\frac{1}{\lambda t}-\frac{2}{t}\right) \mathcal{H}^{2}-\frac{1}{\lambda^{3} t^{2}}=0
$$

This is a generalised Riccati equation which is linearised by the transformation $\mathcal{H}(t)=$ $w^{\prime}(t) / w(t)$, where $w(t)$ satisfies the third order equation

$$
\lambda^{3} t^{2} w^{\prime \prime \prime}+\lambda^{2} t(1-2 \lambda) w^{\prime \prime}-w=0
$$

Now, this equation can be explicitly solved by Frobenius series. However, to obtain the asymptotic behaviour as $\lambda \rightarrow 0$ would require the asymptotic analysis of the resulting series. These series are not conveniently available in the literature. Furthermore, doing the asymptotics this way involves a lot of unnecessary work as a large part of the asymptotic expression of $w(t)$ cancels upon back-substitution to get $\mathcal{H}(t)$. Thus, in order to avoid this unnecessary work, we develop a method of analyzing the Riccati equation directly based on the method of dominant balance. This latter method also generalises to non-linear equations that cannot be linearised, as appears to be the case for column-convex polygons [18].

\section{Formal Perturbation Theory}

One can use the functional equations (3.11), (3.13), (3.14), and (3.25) to derive the critical exponents for these models via singular perturbation theory around $q=1$. We will describe this method in detail for staircase polygons, and then apply it to directed column convex polygons.

The method rests on two assumptions. Initially, it is assumed that an asymptotic expansion in $\epsilon=-\log q$ exists. Then, the results of the perturbation expansion are interpreted in terms of a tricritical scaling ansatz [14] in order to get the crossover exponent.

On the other hand, one can study the asymptotics of the semi-continuous limit, which we shall do below. In this limit it turns out that the scaling behaviour can be calculated 
explicitly without the need to resort to additional assumptions. Needless to say, both methods give consistent results. However, as it is not rigorously shown that the semi-continuous limit preserves the critical structure of the generating functions concerned, it is still useful (and instructive) to directly work within the discrete model.

We now turn to the investigation of the critical structure of staircase polygons. Firstly, we note that eqn. (3.14) can be solved explicitly for $q=1$, resulting in the well-known result for the perimeter generating function for $S_{0}(x, y)=S(x, y, 1)$,

$$
S_{0}(x, y)=\frac{1}{2}\left\{1-x-y-\sqrt{1-2 x-2 y+x^{2}-2 x y+y^{2}}\right\} .
$$

Setting $x=y$, we get

$$
S_{0}(x, x)=\frac{1}{2}\{1-2 x-\sqrt{1-4 x}\},
$$

which has a square root singularity at $x_{c}=1 / 4$, leading to an exponent $\gamma_{u}=-1 / 2$.

In order to get information about the critical structure around this singularity for $q<1$, we now assume that $S(x, y, q)$ has an asymptotic expansion in $\epsilon=-\log q$. For practical purposes, we choose an (equivalent) expansion in the variable $\varepsilon=q^{-1}-1$ instead,

$$
S(x, y, q)=\sum_{n=0}^{\infty} S_{n}(x, y) \varepsilon^{n}
$$

and insert this expansion into (3.14). This leads to eqn. (6.1) and, for all $n>0$, to

$$
\begin{aligned}
S_{n}(x, y)= & \left\{1-x-y-2 S_{0}(x, y)\right\}^{-1} \\
& \times\left\{\left(y+S_{0}(x, y)\right) \sum_{m=1}^{n} S_{n-m}^{(m)}(x, y) \frac{x^{m}}{m !}+\sum_{k=1}^{n-1} S_{k}(x, y) \sum_{m=0}^{n-k} S_{n-m-k}^{(m)}(x, y) \frac{x^{m}}{m !}\right\} .
\end{aligned}
$$

If we look at the behaviour of $S_{n}(x, x)$ at $x_{c}=1 / 4$ we see that the prefactor causes a square root divergence at $x_{c}$. Further divergences are caused from the derivatives in the sums, and closer inspection reveals that $S_{n}(x, x)$ diverges with an exponent

$$
\gamma_{u}^{(n)}=\gamma_{u}+n \Delta \quad \text { with } \quad \gamma_{u}=-\frac{1}{2} \quad \text { and } \quad \Delta=\frac{3}{2} .
$$

This constant increase of $\gamma_{u}^{(n)}$ by a gap exponent $\Delta$ can now be interpreted within the setting of tricritical scaling theory, which is assumed to describe the vicinity of the critical point [14]. The assumption of a scaling form,

$$
S(x, x, q) \sim\left(x_{c}-x\right)^{-\gamma_{u}} \hat{s}\left(\varepsilon\left(x_{c}-x\right)^{-1 / \phi}\right) \quad \text { with } \quad \hat{s}(t) \rightarrow 1 \quad \text { for } \quad t \rightarrow 0
$$


implies that

$$
S_{n}(x, x) \sim\left(x_{c}-x\right)^{-\gamma_{u}-n / \phi} \quad \text { for } \quad x \rightarrow x_{c}
$$

so that we can identify

$$
\phi=\frac{1}{\Delta} .
$$

Using the relation $\gamma_{t}=\phi \gamma_{u}$, this leads to a complete set of tricritical exponents for staircase polygons,

$$
\gamma_{u}=-\frac{1}{2} \quad \gamma_{t}=-\frac{1}{3} \quad \phi=\frac{2}{3} \quad .
$$

Applied to bar-graph polygons, this method gives identical results. The situation is a little more complicated for directed row-convex polygons, as we have to deal with two coupled equations. Firstly, setting $q=1$ gives the perimeter generating function $D_{0}(x, y)=D(x, y, 1)$ as well as $E_{0}(x, y)=D_{\mu}(x, y, 1)$ as solution of

$$
\begin{aligned}
& D_{0}=\left\{1+E_{0}\right\} x\left\{y+D_{0}\right\}+D_{0}\left\{y+D_{0}\right\} \\
& E_{0}=\left\{1+E_{0}\right\} q x\left\{y+E_{0}\right\}+D_{0}\left\{y+E_{0}\right\}+E_{0} y .
\end{aligned}
$$

This can be further reduced to cubic equations for $D_{0}$ and $E_{0}$,

$$
\begin{aligned}
& 0=D_{0}^{3}+2(y-1) D_{0}^{2}+(y-1)(y+x-1) D_{0}+(y-1) y x \\
& 0=x E_{0}^{3}+(y+2) x E_{0}^{2}+\left(2 x y-y^{2}+2 y+x-1\right) E_{0}+y x .
\end{aligned}
$$

$D_{0}(x, x)$ has a square root singularity at

$$
x_{c}=\frac{\sqrt[3]{100}-4}{3} \approx 0.21386
$$

implying that like for staircase polygons,

$$
\gamma_{u}=-\frac{1}{2}
$$

holds. We now apply the above described method to compute the crossover exponent $\phi$. Expanding to first order in $\epsilon=-\log q$ we write

$$
\begin{aligned}
D(x, y, q) & =D_{0}(x, y)+\epsilon D_{1}(x, y) \\
D_{\mu}(x, y, q) & =E_{0}(x, y)+\epsilon E_{1}(x, y) \\
D(q x, y, q) & =D_{0}(x, y)+\epsilon\left(D_{1}(x, y)-x \frac{\partial}{\partial x} D_{0}(x, y)\right) \\
D_{\mu}(x, y, q) & =E_{0}(x, y)+\epsilon\left(E_{1}(x, y)-x \frac{\partial}{\partial x} E_{0}(x, y)\right),
\end{aligned}
$$


which results in the set of equations

$$
\begin{aligned}
D_{1}+x \frac{\partial}{\partial x} D_{0}= & \left(1+E_{0}\right) x\left(D_{1}+x \frac{\partial}{\partial x} D_{0}\right) \\
& +E_{1} x\left(y+D_{0}\right)+D_{0}\left(D_{1}+x \frac{\partial}{\partial x} D_{0}\right)+D_{1}\left(y+D_{0}\right) \\
E_{1}+x \frac{\partial}{\partial x} E_{0}= & \left(1+E_{0}\right) x\left(E_{1}+x \frac{\partial}{\partial x} E_{0}\right) \\
& +E_{1} x\left(y+E_{0}\right)+D_{0}\left(E_{1}+x \frac{\partial}{\partial x} E_{0}\right)+D_{1}\left(y+E_{0}\right)+E_{1} y .
\end{aligned}
$$

These equations are linear in $D_{1}$ and $E_{1}$. On solving these equations for $D_{1}$ and $E_{1}$ one can see on closer inspection that the determinant in the denominator gives a square root divergence and the derivatives of $D_{0}$ and $E_{0}$ in the numerator give an additional exponent increase of 1 , similar to the behaviour of eqn. (6.4) above. Alternatively, inserting the expressions for $D_{0}$ and $E_{0}$ gives the following cubic equation for $D_{1}(x, x)$

$$
\begin{aligned}
0 & =(x-1)\left(x^{4}+4 x^{3}+6 x^{2}-4 x+1\right) x^{2} \\
& -(x-1)\left(x^{7}+8 x^{6}+31 x^{5}+54 x^{4}+34 x^{3}-27 x^{2}+8 x-1\right) D_{1} \\
& +(x-1)\left(3 x^{3}+12 x^{2}+16 x-4\right)\left(x^{3}+3 x^{2}+6 x-2\right) D_{1}^{2} \\
& -\left(3 x^{3}+12 x^{2}+16 x-4\right)^{2} D_{1}^{3} .
\end{aligned}
$$

This implies that $D_{1}(x, x)$ diverges at $x_{c}$ with an exponent of $\gamma_{u}^{(1)}=1$, leading to a gap exponent $\Delta=3 / 2$ which in turn gives the same set of tricritical exponents for directed row-convex polygons as for staircase polygons,

$$
\gamma_{u}=-\frac{1}{2} \quad \gamma_{t}=-\frac{1}{3} \quad \phi=\frac{2}{3}
$$

with the only difference being a shift in the location $x_{c}$ of the phase transition.

\section{Asymptotic Analysis}

For the staircase model the explicit solution of the semi-continuous model and the subsequent asymptotic analysis is possible because of two, equally important, factors. Firstly, the differential equation can be linearised and secondly, the asymptotic forms of the solutions (i.e. Bessel functions) to the linear equation can be readily found in the literature. Unfortunately this not possible with the directed column-convex or more complex models. Thus we develop 
a technique that works directly with the non-linear equation. The technique uses the method of dominant balance [19], similar to that used in WKB calculations.

We use the dominant balance method and see that the same result is obtained as from the asymptotics of the explicit solution [18]. Not only does this provide a check on the method but is also sheds light on the uniformity of the dominant balance result. The dominant balance method is then applied to the directed column-convex model.

\section{Staircase model}

We can now recall [18] the asymptotic behaviour of the staircase generating function (5.17) in the limit $\lambda \rightarrow 0$. This corresponds to an asymptotic behaviour of the Bessel function. Using Olver's result [25] gives

$$
\hat{\mathcal{S}} \sim \frac{1}{2}+\left(\frac{1-4 t}{4 \zeta}\right)^{\frac{1}{2}} \lambda^{\frac{1}{3}} \frac{\operatorname{Ai}^{\prime}\left(\lambda^{-\frac{2}{3}} \zeta\right)}{\operatorname{Ai}\left(\lambda^{-\frac{2}{3}} \zeta\right)} \quad \lambda \rightarrow 0
$$

where

$$
\frac{2}{3} \zeta^{\frac{3}{2}}=\log \left(\frac{1+\sqrt{1-4 t}}{2 \sqrt{t}}\right)-\sqrt{1-4 t} .
$$

As shown by Olver this result is certainly uniform for $t>0$. In the neighbourhood of $t=1 / 4$,

$$
\zeta \sim 2^{-2 / 3}(1-4 t)
$$

and thus (7.1) becomes

$$
\hat{\mathcal{S}} \sim \frac{1}{2}+\left\{\frac{\lambda}{4}\right\}^{\frac{1}{3}} \frac{\operatorname{Ai}^{\prime}\left(\left\{\frac{\lambda}{4}\right\}^{-\frac{2}{3}}\left\{\frac{1}{4}-t\right\}\right)}{\operatorname{Ai}\left(\left\{\frac{\lambda}{4}\right\}^{-\frac{2}{3}}\left\{\frac{1}{4}-t\right\}\right)} \quad \lambda \rightarrow 0 .
$$

Note, (7.4) is no longer uniform for all $t>0$, as was (7.1), but only applies in the neighbourhood of $t=1 / 4$, which is a turning point. Comparing (7.4) with the scaling form (1.6) shows that

$$
f(z)=\frac{\operatorname{Ai}^{\prime}(z)}{\operatorname{Ai}(z)} \quad \gamma_{t}=-\frac{1}{3} \quad \phi=\frac{2}{3} .
$$

The uniformity of the result (7.4) is important as it allows us to interchange the asymptotic limits $\lambda \rightarrow 0$ and $t \rightarrow 1 / 4$. Interchanging the limits shows the asymptotic behaviour (7.4) is consistent with (5.12), as is seen by using the result

$$
\frac{\operatorname{Ai}^{\prime}(x)}{\operatorname{Ai}(x)} \sim-x^{\frac{1}{2}} \quad x \rightarrow \infty
$$


We now rederive the asymptotic behaviour directly from the differential equation using dominant balance. The technique consists of three stages. Firstly a change of variables is made to shift the singular point to origin, then we look for a generalised homogeneous or scaling solution to the differential equation which, finally, is obtained asymptotically using dominant balance.

Thus, for the staircase model the singular point occurs at $t=1 / 4$ where $\hat{\mathcal{S}}=1 / 2$ and so letting $4 s=1-4 t$ and $\hat{\mathcal{S}}=\tilde{\mathcal{S}}-1 / 2$, and substituting into (5.10) gives

$$
\lambda\left(\frac{1}{4}-s\right) \frac{\partial}{\partial s} \tilde{\mathcal{S}}=\tilde{\mathcal{S}}^{2}-s .
$$

Now we look for a generalised homogeneous solution by scaling both the dependent and independent variables. Trying $s=\lambda^{\phi} \bar{s}$ and $\tilde{\mathcal{S}}=\lambda^{\theta} \overline{\mathcal{S}}$ gives

$$
\lambda^{1-\phi+\theta}\left(\frac{1}{4}-\lambda^{\phi} \bar{s}\right) \frac{\partial}{\partial \bar{s}} \overline{\mathcal{S}}=\lambda^{2 \theta} \overline{\mathcal{S}}^{2}-\lambda^{\phi} \bar{s}
$$

A simple analysis shows there are no real values of $\phi$ and $\theta$ which would provide a generalised homogeneous solution. However as $\lambda \rightarrow 0$, the equation is asymptotically dominated by those terms for which the exponent of $\lambda$ is smallest. Thus we look for values of $\phi$ or $\theta$ which give rise to terms having an equally smallest exponent of $\lambda$. In general there may be several different values of $\phi$ and $\theta$ that satisfy this condition. In this situation we choose that solution which asymptotically matches the $\lambda=0$ solution.

Thus, for (7.8) there are the four terms with exponents

$$
r_{1}=1+\theta-\phi \quad r_{2}=1+\theta \quad r_{3}=2 \theta \quad r_{4}=\phi .
$$

Having all four exponents equal (ie. $r_{1}=r_{2}=r_{3}=r_{4}$ ) produces an inconsistent set of equations, we thus look for sets of three equal exponents. Inserting $r_{1}=r_{3}=r_{4}$ produces the solution

$$
\phi=\frac{2}{3} \quad \theta=\frac{1}{3}
$$

As will be shown below, this solution leads to the correct asymptotic matching. Furthermore the values of the exponents are $r_{1}=r_{3}=r_{4}=2 / 3$ and $r_{2}=4 / 3$, thus the elements of the set $\left\{r_{1}, r_{3}, r_{4}\right\}$ are equally smallest. Hence by the principle of dominant balance the term corresponding to $r_{2}$ may be dropped as it does not contribute to the dominant asymptotic 
behaviour. We are thus left with the $\lambda$ independent equation

$$
\frac{1}{4} \frac{\partial}{\partial \bar{s}} \overline{\mathcal{S}}_{0}=\overline{\mathcal{S}}_{0}^{2}-\bar{s}
$$

for the asymptotically dominant part of $\overline{\mathcal{S}}, \overline{\mathcal{S}}_{0}$. Note, we have divided out the common factor of $\lambda^{2 / 3}$. Equation (7.11) is another Riccati equation which is linearised by the transformation

$$
\overline{\mathcal{S}}_{0}=-z^{\prime}(\bar{s}) / 4 z(\bar{s})
$$

if $z(\bar{s})$ satisfies

$$
\frac{d^{2} z}{d \bar{s}^{2}}-16 \bar{s} z=0
$$

A further change of independent variable to $\bar{s}=16^{-1 / 3} \tilde{s}$ gives an Airy equation

$$
\frac{d^{2} z}{d \tilde{s}^{2}}=\tilde{s} z
$$

Hence, the general solution is

$$
\hat{\mathcal{S}} \sim \frac{1}{2}-\left\{\frac{\lambda}{4}\right\}^{\frac{1}{3}} \frac{\mathrm{Ai}^{\prime}\left(\left\{\frac{\lambda}{4}\right\}^{-\frac{2}{3}}\left\{\frac{1}{4}-t\right\}\right)+c \mathrm{Bi}^{\prime}\left(\left\{\frac{\lambda}{4}\right\}^{-\frac{2}{3}}\left\{\frac{1}{4}-t\right\}\right)}{\operatorname{Ai}\left(\left\{\frac{\lambda}{4}\right\}^{-\frac{2}{3}}\left\{\frac{1}{4}-t\right\}\right)+c \operatorname{Bi}\left(\left\{\frac{\lambda}{4}\right\}^{-\frac{2}{3}}\left\{\frac{1}{4}-t\right\}\right)} \quad \lambda \rightarrow 0
$$

where $c$ is an arbitrary constant. Now, this form must asymptotically match (5.12). This is only possible if $c=0$, giving

$$
\hat{\mathcal{S}} \sim \frac{1}{2}-\left\{\frac{\lambda}{4}\right\}^{\frac{1}{3}} \frac{\operatorname{Ai}^{\prime}\left(\left\{\frac{\lambda}{4}\right\}^{-\frac{2}{3}}\left\{\frac{1}{4}-t\right\}\right)}{\operatorname{Ai}\left(\left\{\frac{\lambda}{4}\right\}^{-\frac{2}{3}}\left\{\frac{1}{4}-t\right\}\right)} \quad \lambda \rightarrow 0 .
$$

which is the same result as going via the exact solution. The asymptotic matching of this solution to the $\lambda=0$ solution also shows that the solution $\phi=2 / 3, \theta=1 / 3$ is correct. Note for this choice of $\theta$ and $\phi$ that $\tilde{\mathcal{S}}_{0}=\mathrm{O}(1)$ whilst the order of the remaining term appearing in (7.8) is $\lambda^{2 / 3}$, and hence dropping the $r_{2}$ term is consistent as it is of higher order than the retained terms.

\section{Directed column-convex model}

We now repeat the technique for the directed column-convex model omitting most of the details. First, the singular point is shifted to the origin by $t=\bar{s}+4 / 27$ and $\mathcal{H}=\tilde{\mathcal{H}}-2 / 3$. This is followed by the change of variables to $\bar{s}=\lambda^{\phi} s$ and $\tilde{\mathcal{H}}=\lambda^{\theta} \mathcal{W}$ which results in

$$
\begin{aligned}
& \lambda^{2+\theta-2 \phi}\left(\lambda^{\phi} s+4 / 27\right)^{2} \mathcal{W}^{\prime \prime}-3 \lambda^{1+2 \theta-\phi}\left(\lambda^{\phi} s+4 / 27\right) \mathcal{W} \mathcal{W}^{\prime}+\lambda^{1+\theta-\phi}\left(\lambda^{\phi} s+4 / 27\right) \mathcal{W}^{\prime} \\
& +\lambda^{3 \theta} \mathcal{W}^{3}-\lambda^{2 \theta}(1-\lambda) \mathcal{W}^{2}-\frac{1}{3} \lambda^{1+\theta} \mathcal{W}-\frac{2}{9} \lambda-\lambda^{\phi} s=0
\end{aligned}
$$


If we further assume that $\theta$ and $\phi$ are positive then only five of the exponents can possibly be in a set of equally smallest. The five exponents are

$$
\begin{aligned}
& r_{1}=2+\theta-2 \phi \\
& r_{2}=1+\theta-\phi \\
& r_{3}=2 \theta \\
& r_{4}=\phi \\
& r_{5}=1 .
\end{aligned}
$$

We now systematically search for sets of equally smallest exponents. One set is $r_{2}=r_{3}=$ $r_{4}$. Solving this set of equations gives the same result as the staircase model, ie

$$
\phi=\frac{2}{3} \quad \theta=\frac{1}{3} .
$$

Omitting the seven subdominant terms in (7.17) gives the Riccati equation

$$
\frac{4}{27} \overline{\mathcal{H}}_{0}^{\prime}=\overline{\mathcal{H}}_{0}^{2}+\bar{s}
$$

which again leads to Airy's equation and hence to

$$
\tilde{\mathcal{H}} \sim-\frac{2}{3}+\left\{\frac{4}{27} \lambda\right\}^{\frac{1}{3}} \frac{\operatorname{Ai}^{\prime}\left(\left\{\frac{4}{27} \lambda\right\}^{-\frac{2}{3}}\left\{\frac{4}{27}-t\right\}\right)}{\operatorname{Ai}\left(\left\{\frac{4}{27} \lambda\right\}^{-\frac{2}{3}}\left\{\frac{4}{27}-t\right\}\right)} \quad \lambda \rightarrow 0 .
$$

This shows once again that $\gamma_{t}=-1 / 3$ and $\phi=2 / 3$. Taking the limit $\lambda \rightarrow 0$ gives the result (5.22) confirming that (7.18) is the correct choice.

\section{Conclusion}

We conclude by summarising the principal results of the paper: We have derived non-linear functional equations for the generating functions of the models illustrated in figure 1 . The method of derivation of these functional equations relies on the process of 'inflation' which, for the polygon models, corresponds to adding an area element to the top of each column of the polygon. We obtain $q$-series solutions by linearising the equations. Additionally we take the continuum limit to obtain Riccati differential equations for the corresponding semicontinuous models. These equations can be transformed to linear differential equations and hence solved. 
We have also studied the asymptotics of the generating functions in the neighbourhood of the critical point. This has been done for the discrete models by using a formal perturbation expansion of the non-linear functional equations and for the semi-continuous models by using the method of dominant balance. The asymptotic forms obtained show that all the models of figure 1 belong to the same universality class.

\section{Acknowledgments}

We would like to thank W. Wood for very fruitful discussions and A. L. Owczarek, A.J.G. Guttmann, and C. Pisani for carefully reading the manuscript. We would like to thank the Australian Research Council for financial support.

\section{References}

[1] M. Delest. J. Math Chem, 8:3-18, 1991.

[2] M. Delest and G. Viennot. Theo. Comp. Sc., 34:169-2068, 1984.

[3] M. P. Schützenberger. In Proc. Symp. on Mathematical Theory of Automata, Lecture notes in Computer Science, pages 246-264. Polytechnic Institute of Brooklyn, 1962.

[4] H. N. V. Temperley. Proc. Camb. Phil. Soc., 48:638, 1952.

[5] R. Brak and A. J. Guttmann. J. Phys A., 23:4581, 1990.

[6] R. Brak, A. Guttmann, and S Whittington. J. Phys. A, 25:2437, 1992.

[7] A. L. Owczarek and T. Prellberg. J. Stat. Phys., 70:1175-1194, 1993.

[8] A. L. Owczarek, T. Prellberg, and R. Brak. J. Stat. Phys., 72:737-772, 1993.

[9] P. M. Binder, A. L. Owczarek, A. R. Veal, and J. M. Yeomans. J. Phys. A, 23:L975, 1990.

[10] F. H. Jackson. Trans. Roy. Soc. Edin., 41:1-28, 1903.

[11] M. Rahman. J. Math Analysis and Applications, 125:58-71, 1987. 
[12] G. Gasper and M. Rahman. Basic Hypergeometric Series. Camb. Univ., 1990.

[13] M. P. Delest and J. M. Fedou. In P. Deransart and M. Jourdan, editors, Proc. Workshop on Attribute grammars, pages 46-60. 1990.

[14] R. Brak, A. L. Owczarek, and T. Prellberg. J. Phys. A., 26:4565, 1993.

[15] E Hille. Functional Analysis and Semi-groups, Am. Math. Soc. Colloq. Publ. No 31. American Mathematical Society, New York, 1948.

[16] R. Zwanzig and J. I. Lauritzen, Jr. J. Chem. Phys., 48:3351, 1968.

[17] H. T. Davis. Introduction to non-linear differential and integral equations. Dover, New York, 1962.

[18] R. Brak, A. L. Owczarek, and T. Prellberg. submitted to J. Stat. Phys., 1994.

[19] C. M . Bender and S. A. Orszag. Advanced Mathematical Methods for Scientists and Engineers. McGraw-Hill, Singapore, 1978.

[20] J. M. Fédou and I. Dutour. University of Bordeaux Preprint, 1994.

[21] M. P. Delest and S. Dulucq. Croatica Chemica Acta, 66:59-80, 1993.

[22] M. Bousquet-Melou. University of Bordeaux Preprint, 1993.

[23] X. G. Viennot. private communication.

[24] M. Bousquet-Melou and J. M. Fédou. University of Bordeaux Preprint, 1994.

[25] F. W. J. Olver. Asymptotics and Special Functions. Academic Press, London, 1974. 